\title{
OP auf dem Parkplatz
}

\author{
Mobile OP-Einheiten Wenn der OP-Bereich umgebaut wird, ist die Patientenversorgung häufig \\ nur eingeschränkt oder gar nicht gewährleistet. Abhilfe schaffen mobile OP-Einheiten, die mit \\ modernster Medizintechnik ausgestattet sind. Doch welche organisatorischen, technischen, \\ logistischen und hygienischen Anforderungen sind bei einem solchen Projekt zu bedenken? Lesen Sie, \\ welche Erfahrungen insbesondere in England mit den mobilen OP-Einheiten bisher gemacht wurden.
}

- Thomas Pichote -

Der OP-Bereich eines Krankenhauses muss umgebaut werden. Die Patientenversorgung und der OP-Betrieb dürfen in dieser Zeit keinesfalls unterbrochen werden. Das Klinikpersonal soll aber auch nicht mit zusätzlicher Arbeit oder Überstunden belastet werden.

Um diese Ziele zu erreichen, gibt es nun auch in Deutschland Lösungen mit mobilen OP-Einheiten, die bereits seit 14 Jahren in England erfolgreich an jedem zweiten Krankenhaus genutzt werden. Die mit klinikeigenem und/oder fremdem Equipment komplett ausgestatteten Module werden temporär direkt an einer Klinik platziert und an die Ver- und Entsorgungssysteme des Krankenhauses angeschlossen.

Somit können alle Operationen über Wochen und Monate während einer Bauphase weitergeführt oder für zusätzlich notwendige OP-Kapazitäten genutzt werden.

\section{Gefürchtetes Szenario}

Dass OP-Säle in einer Klinik ausfallen, ist ein bei Verwaltungsdirektoren, Chefärzten und OP-Koordinatoren gefürchtetes Szenario. Wenn diese Situation kurzfristig wegen technischer Defekte eintritt oder geplant wegen eines Umbaus, der Renovierung oder der Erneuerung von Technik, sind alternative Lösungen notwendig.

Keinesfalls sollen geplante OP-Termine abgesagt werden. Sonst könnten möglicherweise Patienten, wegen der Dringlichkeit der OP, die Klinik wechseln. Eine OP-Schließung könnte auch zu Engpässen bei der Versorgung von Patienten führen, die der Rettungsdienst Tag und Nacht in die Klinik bringt. Für den Klinikbetreiber käme es in dieser Zeit zu erheblichen Einnahmeausfällen.

Wesentlich angenehmer ist dagegen die Vorstellung, wenn die OP-Kapazitäten für einen gewissen Zeitraum erweitert werden sollen.

Mobile OP-Einheiten bieten jetzt auch in Deutschland für beide Szenarien qualifizierte Lösungen, den gesamten OP-Betrieb unterbrechungsfrei aufrechtzuhalten.

\section{Planung und Projektierung}

Für die gesamten Planungen ist es ratsam, ein Projektteam zu bilden, bestehend aus

- dem technischem Leiter des Krankenhauses,

- dem Chefarzt der Chirurgie,

- dem OP-Koordinator,

- der Administration der Klinikgruppe

- sowie erforderlichen externen Experten.

Einhergehend mit den Planungen für die Sanierung eines OP-Bereichs sind die Alternativen für Ersatzräume auch aus finanzieller Sicht auszuloten.

Sollen beispielsweise zwei von fünf OPRäumen saniert werden, sind die Kosten und Einnahmen bei der Finanzplanung zu ermitteln, die vom Krankenhausbetreiber für den Ersatzbetrieb samt Personal und den geliehenen mobilen OP-Einheiten für den Ausfall der beiden OP-Säle in der Umbauzeit aufzubringen sind.

Allerdings können durch die Verlagerung der Operationen in die mobilen Einheiten natürlich die Bauarbeiten viel schneller abgeschlossen werden. Sobald die Budgets für die Sanierung freigegeben sind, kann mit der Detailplanung für die Ersatzräume und des darin stattfindenden OP-Betriebs begonnen werden.

\section{Mit dem Tieflader zur Klinik}

Für das temporäre Überbrücken von OPAusfällen gibt es in Deutschland Lösungen mit mobilen OP-Einheiten der Firma Vanguard Healthcare ( $\rightarrow$ Abb. 1 bis 7). Dazu hat der englische Marktführer fertig eingerichtete
OP-Module konzipiert, die auf einem Tieflader samt Lkw-Zugmaschine in etwa einem Tag deutschlandweit zu einer Klinik gefahren werden und dort nach weiteren etwa vier Stunden betriebsbereit stehen können.

Am englischen Princess Royal Hospital in Telford wurden zusätzliche Kapazitäten für endoskopische Eingriffe während der Modernisierung der Abteilung benötigt. Dazu stand eine mobile OP-Einheit, die einen Gesamtwert von über einer Million Euro hat, für sechs Wochen an der Klinik.

„Ich hatte den Eindruck, als ob wir eine Koloskopie in einem Wohnwagen auf einem Parkplatz durchführen würden. Aber tatsächlich ist die mobile Einheit innen viel größer, als sie einem zunächst erscheint. Die technischen Anlagen in den mobilen Einheiten sind sehr modern. Praktisch handelte es sich um eine sehr zufriedenstellende und auf dem neuesten Stand der Technik stehende Anlage“, sagt Mark Cheetham, Medizinischer Direktor der Shrewsburyund Telford-Klinikgruppe.

„Wir haben vorher zusammen mit dem OP-Management der Klinik den Einsatz unserer mobilen OP-Einheit geplant und alle technischen Anforderungen auch mit der technischen Leitung des Krankenhauses abgestimmt“, erklärt Thomas Pichote, Deutschland Manager von Vanguard Healthcare.

Die 28 Tonnen schwere OP-Einheit wurde am frühen Abend mit einem Tieflader angeliefert. Nach dem Ausfahren von vier Stützen stand die gesamte Einheit frei auf dem Besucherparkplatz und die Zugmaschine zog den leeren Tieflader-Auflieger weg. Mit den Stützen wurde der Fußboden der OP-Einheit entsprechend dem dortigen Untergrund in der Waagerechten nivelliert und die Seitenwände herausgezogen.

Nach dem Anbringen der beiden Rampen für den Ein- und Ausgang sowie dem 
Anschluss an die dort vorbereiteten Versorgungsleitungen für Strom (125 Ampere), Frisch- und Abwasser sowie die Telekommunikation (Telefon, Netzwerk mit Internet der Klinik) durch Servicemitarbeiter des Krankenhauses, war die OP-Einheit innerhalb von einem halben Tag einsatzbereit.

Danach wurde die übliche OP-Ausstattung der Klinik in die Räume gebracht und in die Schränke zusammen mit einem erfahrenen Mitarbeiter des Modulbetreibers eingeräumt. Nach einer Einweisung durch einen Mitarbeiter des Herstellers und einem Test der Technik, hat der Reinigungsservice die Innenräume desinfiziert.

Binnen 24 Stunden war die Operationsbereitschaft hergestellt. Als am nächsten Morgen die Handwerker den OP-Trakt in der Klinik in Beschlag genommen hatten, wurde zeitgleich der erste Patient auf einer Liege vom Klinik-Transportdienst in den OP-Vorbereitungsraum der Einheit geschoben.

\section{Räume und Ausstattung}

Die mobile OP-Einheit besteht aus fünf Räumen: Personalumkleide, Vorbereitungsraum, OP, Aufwach- und Materialraum.

„Wir bieten den Ärzten sowie den OPund Pflegekräften bei Nutzung ihrer vertrauten Ausstattung äquivalente Räume, nur auf einer etwas kleineren Fläche. Die Qualität der medizinischen und pflegerischen Leistungen kann auf dem gleichen Niveau gehalten werden - teilweise kann sie sogar durch bessere Klimatechnik und dem Laminar-Air-Flow noch gesteigert werden - wie im festen Klinikgebäude“, sagt Pichote. Die Innenräume sind auch bei arktischen und tropischen Außentemperaturen angenehm klimatisiert.
„Unsere mobilen OP-Einheiten verfügen über alle in Europa vorgeschriebenen Sicherheitseinrichtungen wie Brandschutz, Einbruch und Fernüberwachung sowie über die technischen Standards nach den jeweils länderspezifischen Vorgaben und sind bei einem etwaigen Schadenfall versichert“, ergänzt der Deutschland Manager.

\section{Arbeiten in der mobilen OP-Einheit}

„Aufgrund des enormen Umfangs der Bauarbeiten mussten wir unser Hauptgebäude für einige Monate räumen. Eine weitere Voraussetzung für die Erteilung der JAG-Zertifizierung (britisches Zertifizierungssystem für Endoskopie-Abteilungen) ist auch die Pünktlichkeit der Durchführung von endoskopischen Eingriffen. Daher waren wir sehr daran interessiert, dass wir während dieser Zeit auch wirklich alle Eingriffe durchführen konnten“, erinnert sich Cheetham.

„Eine mobile Einheit an unserem Standort aufzubauen bedeutete, dass wir viele unserer endoskopischen Eingriffe dorthin verlagern und auch während der Bauarbeiten zusätzliche Eingriffe in der mobilen Einheit durchführen konnten. Mit diesen Mitteln und Möglichkeiten, die für uns besonders wichtig waren, konnten wir auch das Hauptgebäude komplett räumen. Somit hatten die Bauleute freie Hand, um ihre Arbeiten ordnungsgemäß durchzuführen.“

\section{Feedback vom Personal}

„Zuerst waren alle sehr skeptisch. Tatsächlich ist das aber ein sehr angenehmer Arbeitsplatz. Wir befinden uns in einem Operationssaal, der ein eigenständiges mobiles OP-Zentrum bildet. Dieser Bereich befindet sich abgeschirmt von der hekti- schen Notfallaufnahme. Unser Klinikpersonal hat schon gefragt, ob wir die OP-Einheit das ganze Jahr lang behalten können. Ich meine, das zeigt doch eindrucksvoll, dass das Arbeitsumfeld dort sehr angenehm ist", sagt Cheetham.

\section{Abtransport der mobilen OP-Einheit}

Als der renovierte OP-Trakt in der Klinik nach 17 Wochen fertiggestellt und gereinigt war, musste das Personal samt dem Klinikmaterial aus der liebgewonnenen mobilen OP-Einheit wieder ausziehen. Dazu wurden zunächst alle Ver- und Entsorgungsleitungen vom Krankenhaus an der Einheit entfernt.

Dann rückte wieder eine Lkw-Zugmaschine samt leerem Tieflader-Auflieger an und fuhr rückwärts unter die mobile OP-Einheit. Die Seitenwände wurden eingezogen und die Stützen eingeklappt. Abschließend fuhr der Lastwagen vom Klinikgelände zu seinem nächsten Einsatzort.

\section{Interessenkonflikt}

Thomas Pichote ist Angestellter der Firma Vanguard Healthcare Solutions.

\section{Autor}

Thomas Pichote

Country Manager Deutschland für Vanguard Healthcare Solutions E-Mail: thomaspichote@vanguardhs.com

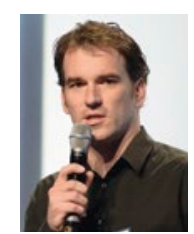

\section{Bibliografie}

DOI 10.1055/s-0042-118496 Im OP 2017; 7: 6-8

(c) Georg Thieme Verlag KG

Stuttgart · New York · ISSN 1611-7905

Fazit

Mobile OP-Einheiten, wie sie seit 14 Jahren in England erfolgreich an jedem zweiten Krankenhaus genutzt werden, bieten nun auch in Deutschland äquivalente Arbeitsbedingungen und die gewohnte Ausstattung, wie sie in gleicher Form in der Klinik vorhanden ist.

Eine frühzeitige Planung und Beratung durch externe Experten vor einem Umbau, der Renovierung oder der Erneuerung von Technik ist ratsam. Dann stellt die mobile OP-Einheit eine effiziente und alternative Lösung dar.

Damit können OP-Programme unterbrechungsfrei fortgesetzt werden. Infolgedessen kommt es zu keinen Einnahmeausfällen für den Klinikbetreiber, keinen Überstunden beim Klinikpersonal und kürzeren Bauzeiten im Krankenhaus-OP.

Die Kosten für die Nutzung einer mobilen OP-Einheit sollten in die Kalkulation der Umbauarbeiten mit eingeplant werden. Alle Patienten des Krankenhauses können in den mobilen OP-Einheiten auf dem qualitativ gleichwertigen Niveau operiert, versorgt und betreut werden, wie im Klinikgebäude, nur in kompakteren Räumlichkeiten. 